\title{
Application of magnetic resonance fingerprinting to differentiate grade I transitional and fibrous meningiomas from meningothelial meningiomas
}

\author{
Rui Zhang ${ }^{1,2,3 \#}$, Yu Shen ${ }^{1,2 \#}$, Yan Bai ${ }^{1,2}$, Xianchang Zhang ${ }^{4}$, Wei Wei ${ }^{1,2}$, Ruijuan Lin $^{3}$, Qin Feng ${ }^{1,2}$, \\ Mengke Wang ${ }^{1,2}$, Menghuan Zhang ${ }^{1,2}$, Mathias Nittka ${ }^{5}$, Gregor Koerzdoerfer ${ }^{5}$, Meiyun Wang ${ }^{1,2}$ \\ ${ }^{1}$ Department of Medical Imaging, Zhengzhou University People's Hospital and Henan Provincial People's Hospital, Zhengzhou, China; ${ }^{2}$ Henan \\ Key Laboratory of Neurological Imaging, Zhengzhou, China; ${ }^{3}$ Academy of Medical Sciences, Zhengzhou University, Zhengzhou, China; ${ }^{4}$ MR \\ Collaboration, Siemens Healthcare Ltd., Beijing China; ${ }^{5}$ Magnetic Resonance, Siemens Healthcare, Erlangen, Germany
}

\#These authors contributed equally to this work.

Correspondence to: Meiyun Wang. Department of Medical Imaging, Zhengzhou University People's Hospital and Henan Provincial People's Hospital, No. 7 Weiwu Road, Zhengzhou 450003, China. Email: mywang@ha.edu.cn.

Background: The choice of surgical treatment for meningiomas is affected by the subtype and clinical characteristics. Therefore, an accurate preoperative diagnosis is essential. Current magnetic resonance imaging (MRI) technology is unable to distinguish between meningioma subtypes. In the present study, we compared and evaluated the utility of conventional MRI, magnetic resonance fingerprinting (MRF), and diffusion-weighted imaging (DWI) in differentiating World Health Organization grade I transitional and fibrous meningiomas from meningothelial meningiomas.

Methods: Forty-six patients with pathologically confirmed meningiomas (15 meningothelial, 18 transitional, and 13 fibrous) were enrolled in the present study. All patients underwent conventional MRI, MRF, and DWI scans before surgery using a $3 \mathrm{~T}$ scanner. The Jonckheere-Terpstra test was used to analyze differences in the signal and enhancement characteristics of the three groups from $\mathrm{T}_{1}$-weighted imaging (T1WI) and $\mathrm{T}_{2}$-weighted imaging (T2WI). To investigate the difference in quantitative $\mathrm{T} 1$ and $\mathrm{T} 2$ values derived from MRF and apparent diffusion coefficient (ADC) values between the three groups using the Kruskal-Wallis test, regions of interest (ROIs) were manually drawn on the parenchymal portion of the tumors; $\mathrm{P}<0.017$ was considered statistically significant after Bonferroni correction for multiple comparison. The receiver operating characteristic (ROC) curve was used to evaluate the diagnostic performances of the different parameters.

Results: Meningothelial meningiomas had significantly higher T1 and T2 values than transitional and fibrous meningiomas (all $\mathrm{P}<0.017$ ). ROC analysis results revealed that the combination of $\mathrm{T} 1$ and $\mathrm{T} 2$ values had the largest area under the curve (AUC). The AUC for the combination of T1 and T2 values was 0.826 between meningothelial and transitional meningiomas, and the AUC for the combination of T1 and T2 values between meningothelial and fibrous meningiomas was 0.903 . No significant differences were found in the $\mathrm{T} 1$ and $\mathrm{T} 2$ values between transitional and fibrous meningiomas. There were also no statistically significant differences in the conventional MRI (including T1WI, T2WI, and contrast-enhanced T1WI) and $\mathrm{ADC}$ values between the three meningioma subtypes (all $\mathrm{P}>0.05$ ).

Conclusions: MRF may provide more quantitative information than either conventional MRI or DWI for differentiating transitional and fibrous meningiomas from meningothelial meningiomas. T1 and T2 values derived from MRF may distinguish transitional and fibrous meningiomas from meningothelial meningiomas, and the combination of $\mathrm{T} 1$ and $\mathrm{T} 2$ values provides the highest diagnostic efficacy. 
Keywords: Diffusion-weighted imaging (DWI); magnetic resonance imaging (MRI); magnetic resonance fingerprinting (MRF); meningioma; subtypes

Submitted Jun 09, 2020. Accepted for publication Nov 06, 2020.

doi: $10.21037 /$ qims-20-732

View this article at: http://dx.doi.org/10.21037/qims-20-732

\section{Introduction}

Meningiomas are the second most common primary tumor of the central nervous system (1). According to the 2016 World Health Organization (WHO) classification of tumors of the central nervous system, meningiomas can be classified into 3 grades and 15 pathological subtypes (2); 9 subtypes are classified as WHO grade I, 3 subtypes are classified as WHO grade II and 3 are classified as grade III. Most meningiomas are WHO grade I, and the most common histological subtypes are meningothelial, transitional, and fibrous meningiomas. Although grade I meningiomas are benign (3), patients with these tumors do not always have satisfactory surgical outcomes. Meningothelial meningiomas rarely involve intra-tumoral hemorrhage, whereas transitional and fibrous meningiomas are prone to hemorrhage (4). Therefore, compared with meningothelial subtype, transitional and fibrous meningiomas are associated with a higher bleeding risk during surgery and a worse outcome at follow-up. The accurate diagnosis of transitional and fibrous meningiomas before surgery is therefore essential for selecting the most appropriate surgical procedure.

With the continuous development of technology, the clinical application of imaging techniques, such as magnetic resonance imaging (MRI) and computed tomography, has significantly improved the accuracy of localization and the qualitative diagnosis of meningiomas. However, conventional techniques can only reflect the gross morphological changes of tumors. Most subtypes of meningioma cannot be characterized by conventional imaging, so challenges remain regarding their differentiation before surgery $(5,6)$. Conventional MRI, including $\mathrm{T}_{1}$-weighted imaging (T1WI), $\mathrm{T}_{2}$-weighted imaging (T2WI), and contrast-enhanced T1WI, has been widely used in the preoperative diagnosis of meningiomas. Diffusion-weighted imaging (DWI) is a technique that relies on the movement of water molecules at the cellular level (7). The apparent diffusion coefficient (ADC) value, which is derived from DWI, quantifies the diffusion state of water molecules. Different pathologies alter the movement of water molecules in different ways, and ADC values can allow researchers to differentiate between pathologies, including different tumor types $(8,9)$. However, the ability of conventional MRI and DWI-derived ADC values to differentiate WHO grade I transitional and fibrous meningiomas from meningothelial meningiomas is limited, because of the overlap in imaging characteristics and ADC values (10). Therefore, new MRI techniques are needed to improve diagnostic accuracy in differentiating between meningioma subtypes.

Magnetic resonance fingerprinting (MRF) is a novel MRI technique that can simultaneously estimate multiple quantitative biophysical parameters of different tissue components $(11,12)$. Previous studies have shown that quantitative MRI can better detect physiological and morphological changes, such as liver and cardiac fibrosis, than conventional qualitative MRI $(13,14)$. MRF addresses the limitations of traditional MRI technology, which cannot simultaneously separate and accurately estimate physiological parameters of different tissues, and achieves parallel quantitative imaging of various physiological parameters. The entire MRF process can be divided into three parts: data acquisition, pattern matching, and organizational feature visualization (15). It is a simple, fast, non-invasive quantitative MRI technique that enables multiple organizational characteristics to be acquired simultaneously in a single, efficient acquisition. MRF uses random excitation flip angles and repetition times (TRs) for data acquisition to obtain incoherent and different magnetic resonance time courses (16). In the pattern matching stage of the process, the unique "fingerprints" from each voxel are matched to a set of simulated fingerprints in a dictionary generated by the MRF sequence using the same acquisition parameters. The magnetic resonance parameters that produce the best match are used as definite quantity results (17). MRF has been proved to be a valuable tool for the characterization of pathological conditions in diseases, such as multiple sclerosis (18), epilepsy (19), and brain tumors (17). However, to the best of our knowledge, MRF has not been used for the differential diagnosis of 
meningioma subtypes. In the present study, we aimed to evaluate and compare the ability of MRF, conventional MRI, and DWI in differentiating WHO grade I transitional and fibrous meningiomas from meningothelial meningiomas.

\section{Methods}

\section{Patients}

The present study was approved by the local institutional review boards, and written informed consent was obtained from all participants. From June 2019 to December 2019, 53 patients with suspected meningiomas (36 women and 17 men; age range: $29-72$ years, mean \pm standard deviation age: $55.7 \pm 10.1$ years) were enrolled in the study. The inclusion criteria were as follows: (I) conventional brain MRI scans and enhancements had been performed without preoperative biopsy, radiotherapy, chemotherapy, and other interventions or treatments; (II) postoperative pathological results confirmed the presence of WHO grade I meningiomas, including meningothelial, transitional, and fibrous meningiomas, after MRI examination; and (III) the MRI quality was adequate for further analysis. The exclusion criteria were as follows: (I) recent cerebrovascular diseases or cerebrovascular accidents; and (II) a previous history of other head and neck tumors. After pathological examination, four patients were found not to have meningiomas, and three patients had images with severe motion artifacts. Finally, 46 patients (age range: $31-72$ years, mean \pm standard deviation age: $56.0 \pm 9.6$ years, 32 women and 14 men) with pathologically confirmed meningothelial $(\mathrm{n}=15)$, transitional $(\mathrm{n}=18)$, and fibrous meningiomas $(\mathrm{n}=13)$ were included in the study.

\section{MRI data acquisition}

Before surgery, all patients underwent MRI using a $3 \mathrm{~T}$ MAGNETOM Skyra scanner (Siemens Healthcare, Erlangen, Germany) equipped with a 20-channel head/neck coil. The MRI protocol included the following sequences: a prototype spiral fast imaging with steady-state precession MRF sequence with the same parameters as the reference [field of view $(\mathrm{FOV})=256 \times 256 \mathrm{~mm}^{2}$, matrix $=256 \times 256$, slice thickness $=5 \mathrm{~mm}$, flip angle variable $=0-74^{\circ}$, TR between 12.1 and $15.0 \mathrm{~ms}$, measurements $=3,000$, acquisition time $=12 \mathrm{~min}$ and $18 \mathrm{~s}$, number of slices $=18$ ] and conventional MRI sequences (slice thickness $=5 \mathrm{~mm}$; FOV $=256 \times 256 \mathrm{~mm}^{2}$ ) (12), including axial and sagittal T1WI [TR $=250 \mathrm{~ms}$, echo time $(\mathrm{TE})=2.46 \mathrm{~ms}$ ], axial T2WI (TR: 5,000 ms, TE: $96 \mathrm{~ms}$ ), axial fluid attenuated inversion recovery (TR: 7,500 ms, TE: $81 \mathrm{~ms}$; inversion recovery: 2,300 ms), and DWI sequences (TR $=4,140 \mathrm{~ms}$, TE $=64 \mathrm{~ms}$, $b$ value: 0 and $1,000 \mathrm{~s} / \mathrm{mm}^{2}$ ). The gadolinium-diethylenetriamine pentaacetic acid contrast agent $(0.1 \mathrm{mmol} / \mathrm{kg}$; Xi'an Ruixi Biological Technology Co., Ltd, Xi'an, China) was administered intravenously to perform contrast-enhanced imaging; the above mentioned T1WI scanning parameters were used.

\section{MRI data analysis}

All MRI data were analyzed using a workstation (syngo. via; Siemens Healthcare, Germany). The MRI data were independently analyzed by two radiologists (with 9 and 7 years of experience in diagnostic radiology, respectively), who were blinded to the histological results. For T1WI and T2WI, hyperintense meningiomas were defined as those with a signal intensity that was obviously higher than that of the surrounding gray matter; slightly hyperintense meningiomas were defined as those with a signal intensity that was slightly higher than that of the surrounding gray matter; isointense meningiomas were defined as those with a signal intensity similar to that of the surrounding gray matter; slightly hypointense meningiomas were defined as those with a signal intensity that was slightly lower than that of the surrounding gray matter; and hypointense meningiomas were defined as those with a signal intensity that was obviously lower than that of the surrounding gray matter. Meningioma enhancement levels were evaluated on the contrast-enhanced T1WI images and were compared to the cavernous sinus, which served as a reference. Mild enhancement indicated that the degree of enhancement was less than that in the cavernous sinus. Moderate enhancement indicated that the degree of enhancement was similar to that in the cavernous sinus. Significant enhancement indicated that the degree of enhancement was greater than that in the cavernous sinus.

The ADC map was calculated from DWI. The quantitative $\mathrm{T} 1$ and $\mathrm{T} 2$ maps were generated by matching the measured MRF signal time course to the dictionary. Because the tumor features and contours were clearly displayed by contrast-enhanced T1WI, the regions of interest (ROIs) were manually drawn on the solid tumor components of the contrast-enhanced image using the Siemens syngo.via application, and the areas of hemorrhage and necrosis were excluded. The ROIs drawn on the 

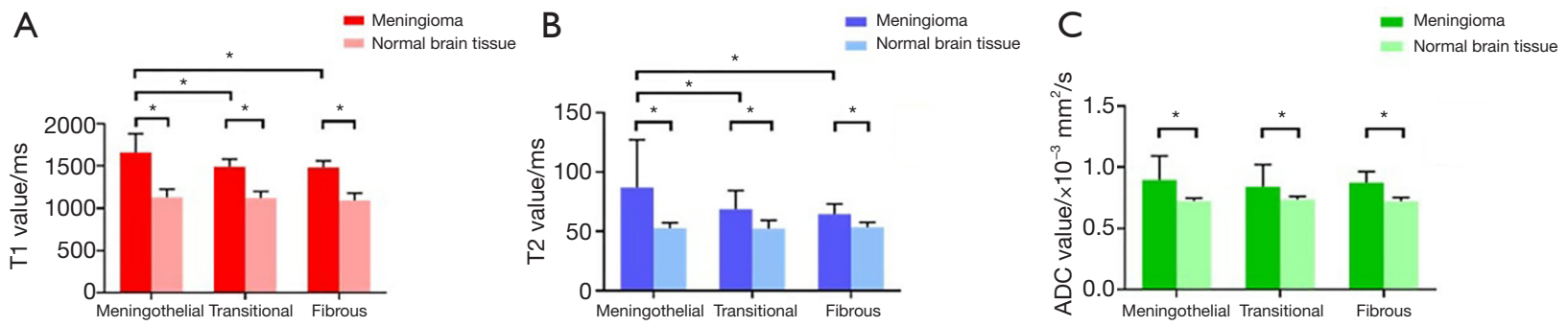

Figure 1 Histograms of quantitative values for three meningioma subtypes. (A) Histogram of T1 values of three different subtypes of meningioma and the contralateral normal brain tissue. There were statistically significant differences in T1 values between meningothelial and transitional or fibrous meningiomas. T1 values between meningioma and contralateral normal brain tissue were statistically different in the three subtypes. (B) Histogram of T2 values of three different subtypes of meningioma and the contralateral normal brain tissue. There were statistically significant differences in T2 values between meningothelial and transitional or fibrous meningiomas. T2 values between meningioma and contralateral normal brain tissue were statistically different in the three subtypes. (C) Histogram of apparent diffusion coefficient (ADC) values of three different subtypes of meningioma and the contralateral normal brain tissue. No statistically significant differences were found in ADC values between the three subtypes. ADC values between meningioma and contralateral normal brain tissue were statistically different in the three subtypes. *Statistical difference between two groups.

enhancement images were then copied to the quantitative T1, T2, and ADC maps to extract tumor characteristics. The T1, T2, and ADC values of normal brain tissue were also included for analysis. Normal brain tissue values were derived from the ROI on the contralateral side, which had a similar volume and location to the tumor ROI. The mean value of each ROI was used for the statistical analysis for each quantitative parameter. All analysis results were reviewed by an expert with extensive experience in the radiological diagnosis of central nervous system diseases.

\section{Statistical analyses}

The differences in T1WI and T2WI signal and enhancement characteristics between different subtypes of meningiomas were analyzed using the Jonckheere-Terpstra test. The Kruskal-Wallis test was used to evaluate the differences in the T1, T2, and ADC values of normal brain tissue and tumor tissue between the transitional, fibrous, and meningothelial subtypes. After Bonferroni correction for multiple comparisons, $\mathrm{P}<0.017$ was considered to show statistical significance. The Two-Related Samples test was used to calculate the difference in $\mathrm{T} 1, \mathrm{~T} 2$, and $\mathrm{ADC}$ values between meningioma and the contralateral normal tissue of the three different tumor subtypes. Receiver operating characteristic (ROC) curve analysis was conducted, and the areas under the ROC curves (AUCs) were calculated between statistically significant groups to evaluate the efficacy of $\mathrm{T} 1$ and $\mathrm{T} 2$ values in differentiating between different subtypes of meningiomas. The diagnostic sensitivity and specificity of the values were calculated, and thresholds were selected to maximize the Youden index. If two or more quantitative parameters were found to be statistically significant, binary logistic regression analysis was used to combine significant parameters to obtain a combined variable. A ROC analysis was performed to evaluate the different diagnostic performances of the combined variables between different subtypes of meningiomas. All statistical analyses were analyzed using SPSS version 18.0 (SPSS, Chicago, IL, USA).

\section{Results}

The T1 values (mean \pm standard deviation) for meningothelial, transitional, and fibrous meningiomas were $1,661 \pm 222,1,491 \pm 91$, and $1,485 \pm 78 \mathrm{~ms}$, respectively. The $\mathrm{T} 2$ values (mean \pm standard deviation) for meningothelial, transitional, and fibrous meningiomas were $87 \pm 40$, $69 \pm 15$, and $65 \pm 9 \mathrm{~ms}$, respectively. As shown in Figure 1, the meningothelial meningiomas had significantly higher T1 $(\mathrm{P}=0.002$ and $\mathrm{P}=0.002$, respectively; significant after Bonferroni correction) and $\mathrm{T} 2$ values $(\mathrm{P}=0.002$ and $\mathrm{P}=0.001$, respectively; significant after Bonferroni correction) than transitional and fibrous meningiomas. No statistically significant difference was found in $\mathrm{T} 1$ and $\mathrm{T} 2$ values between transitional and fibrous meningiomas $(\mathrm{P}=0.936$ and $\mathrm{P}=0.617$, respectively). The ADC values of meningothelial, transitional, and fibrous meningiomas were $(0.89 \pm 0.20) \times 10^{-3}$, 
Table $1 \mathrm{~T}_{1}$-weighted imaging signal characteristics of different meningioma subtypes

\begin{tabular}{lccccc}
\hline Pathological pattern & Hypointense & Slightly hypointense & Isointense & Slightly hyperintense & Hyperintense \\
\hline Meningothelial meningiomas & 2 & 2 & 10 & 1 & 0 \\
Transitional meningiomas & 2 & 5 & 9 & 2 & 0 \\
Fibrous meningiomas & 1 & 3 & 9 & 0 & 0 \\
Summation & 5 & 10 & 28 & 3 & 0 \\
\hline
\end{tabular}

Numbers represent patients with different $\mathrm{T}_{1}$-weighted imaging (T1WI) signal characteristics.

Table $2 \mathrm{~T}_{2}$-weighted imaging signal characteristics of different meningioma subtypes

\begin{tabular}{lccccc}
\hline Pathological pattern & Hypointense & Slightly hypointense & Isointense & Slightly hyperintense & Hyperintense \\
\hline Meningothelial meningiomas & 0 & 1 & 7 & 3 & 4 \\
Transitional meningiomas & 1 & 3 & 11 & 2 & 1 \\
Fibrous meningiomas & 0 & 1 & 9 & 2 & 1 \\
Summation & 1 & 5 & 27 & 7 & 6 \\
\hline
\end{tabular}

Numbers represent patients with different $\mathrm{T}_{2}$-weighted imaging (T2WI) signal characteristics.

Table 3 Magnetic resonance imaging enhancement characteristics of different meningioma subtypes

\begin{tabular}{lcccc}
\hline Pathological pattern & Mild & Moderate & Significant & Summation \\
\hline Meningothelial meningiomas & 3 & 9 & 3 & 15 \\
Transitional meningiomas & 6 & 10 & 7 & 1 \\
Fibrous meningiomas & 5 & 26 & 6 & 13 \\
Summation & 14 & 46 \\
\hline
\end{tabular}

Numbers represent patients with different magnetic resonance imaging (MRI) enhancement characteristics.

$(0.84 \pm 0.18) \times 10^{-3}$, and $(0.87 \pm 0.09) \times 10^{-3} \mathrm{~mm}^{2} / \mathrm{s}$, respectively, but there were no statistical differences between the three groups $(\mathrm{P}=0.258)$. In terms of normal brain tissue, the $\mathrm{T} 1$ values (mean \pm standard deviation) of the meningothelial, transitional, and fibrous subtypes were 1,131 $\pm 94,1,123 \pm 76$, and $1,092 \pm 87 \mathrm{~ms}$, respectively; the T2 values (mean \pm standard deviation) of the meningothelial, transitional, and fibrous subtypes were $53 \pm 5,52 \pm 7$, and $54 \pm 4$ ms, respectively; and the ADC values of the meningothelial, transitional, and fibrous subtypes were $(0.72 \pm 0.02) \times 10^{-3},(0.74 \pm 0.02) \times 10^{-3}$, and $(0.72 \pm 0.03) \times 10^{-3} \mathrm{~mm}^{2} / \mathrm{s}$, respectively. The T1, T2, and ADC values of normal brain tissue were not statistically different between the three subtypes $(\mathrm{P}=0.480, \mathrm{P}=0.646$, and $\mathrm{P}=0.197$, respectively). The $\mathrm{T} 1, \mathrm{~T} 2$, and $\mathrm{ADC}$ values between meningioma and the contralateral normal brain tissue of the three tumor subtypes were statistically different (all $\mathrm{P}<0.05$ ).

Tables 1 and 2 summarize T1WI and T2WI signal characteristics. MRI enhancement characteristics of patients are shown in Table 3. There were no statistical differences in T1WI, T2WI, or contrast-enhanced T1WI between the three meningioma groups $(\mathrm{P}=0.754, \mathrm{P}=0.203$, and $\mathrm{P}=0.202$, respectively). Data from three representative patients with different meningioma subtypes are shown in Figures 2-4. Figure 5 shows the biopsy images of the three representative patients. The T1WI and T2WI signals of these patients were similar, and all showed obvious enhancement. Therefore, there are still limitations in distinguishing between meningioma subtypes using conventional MRI. In the maps generated by MRF, the T1 and T2 values of meningothelial patients appeared to be slightly higher than those of transitional and fibrous patients. Figure 6 shows the ROC curves of the T1, T2, and combined T1 and T2 (combined variable) values between statistically significant groups. 

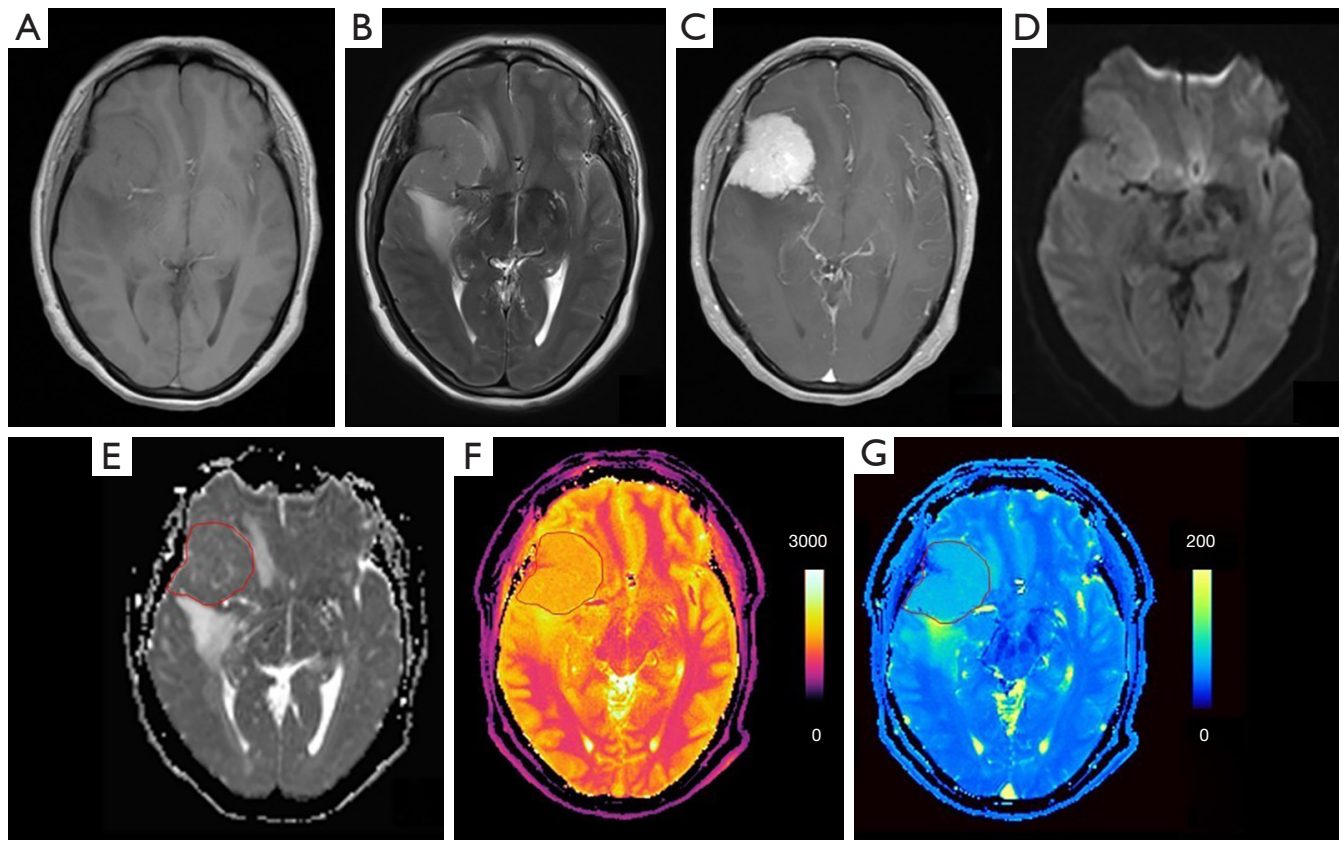

Figure 2 Data from a representative 52-year-old female patient with a meningothelial meningioma. (A) $\mathrm{T}_{1}$-weighted imaging (T1WI) MRI; (B) $\mathrm{T}_{2}$-weighted imaging (T2WI) MRI; (C) contrast-enhanced T1WI; (D) diffusion-weighted imaging (DWI); (E) apparent diffusion coefficient map; (F) T1 value derived from magnetic resonance fingerprinting (MRF); (G) T2 value derived from MRF.
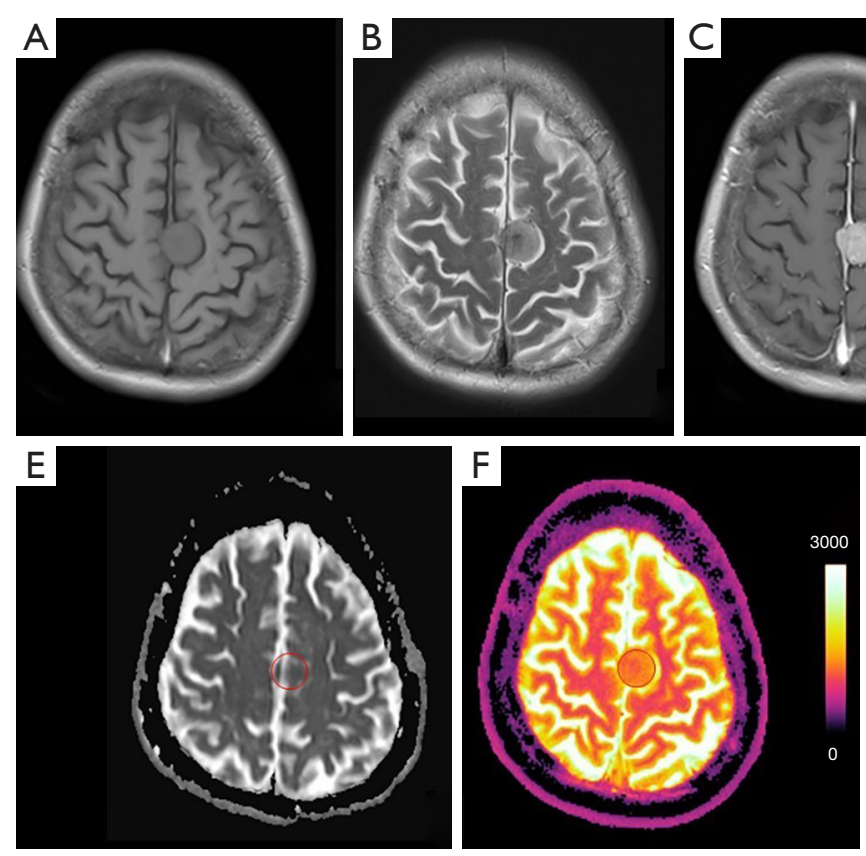
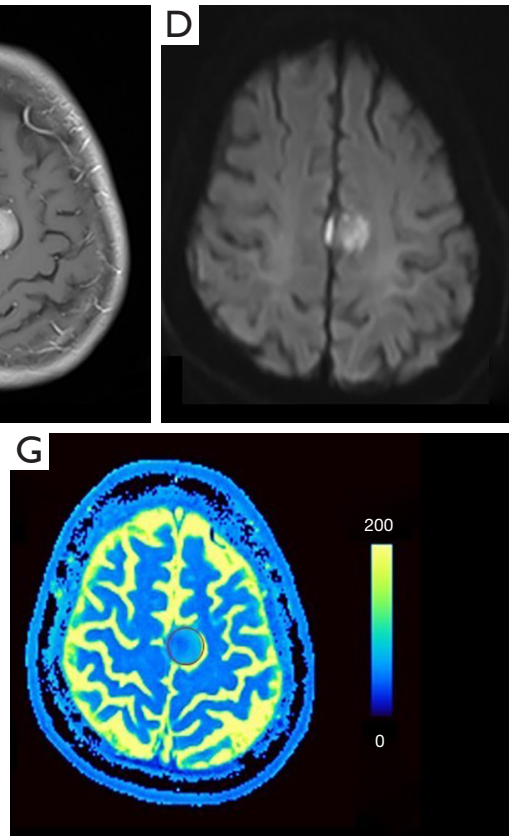

Figure 3 Data from a representative 71-year-old female patient with transitional meningioma. (A) $\mathrm{T}_{1}$-weighted imaging (T1WI) MRI; (B) $\mathrm{T}_{2}$-weighted imaging (T2WI) MRI; (C) contrast-enhanced T1WI; (D) diffusion-weighted imaging (DWI); (E) apparent diffusion coefficient map; (F) T1 value derived from magnetic resonance fingerprinting (MRF); (G) T2 value derived from MRF. 

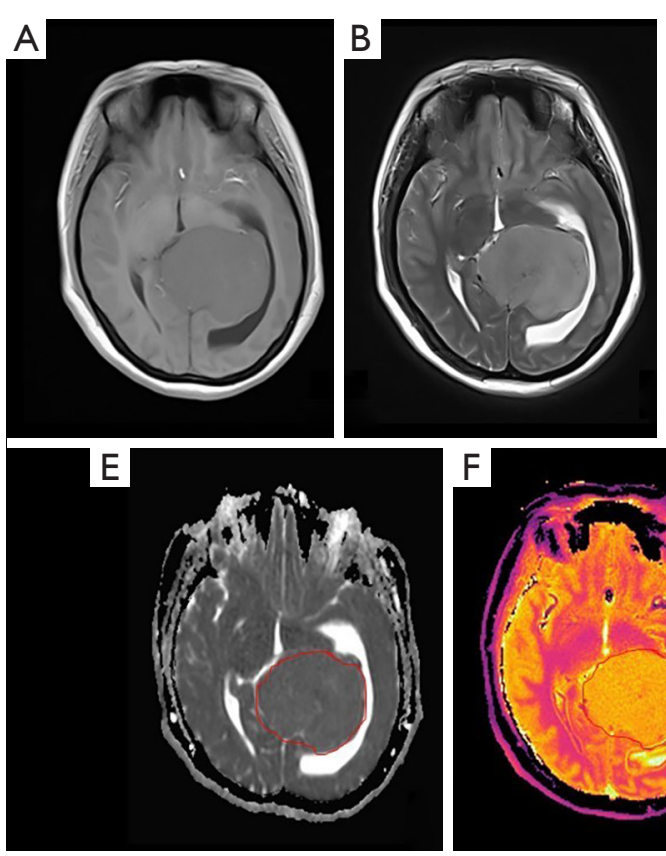
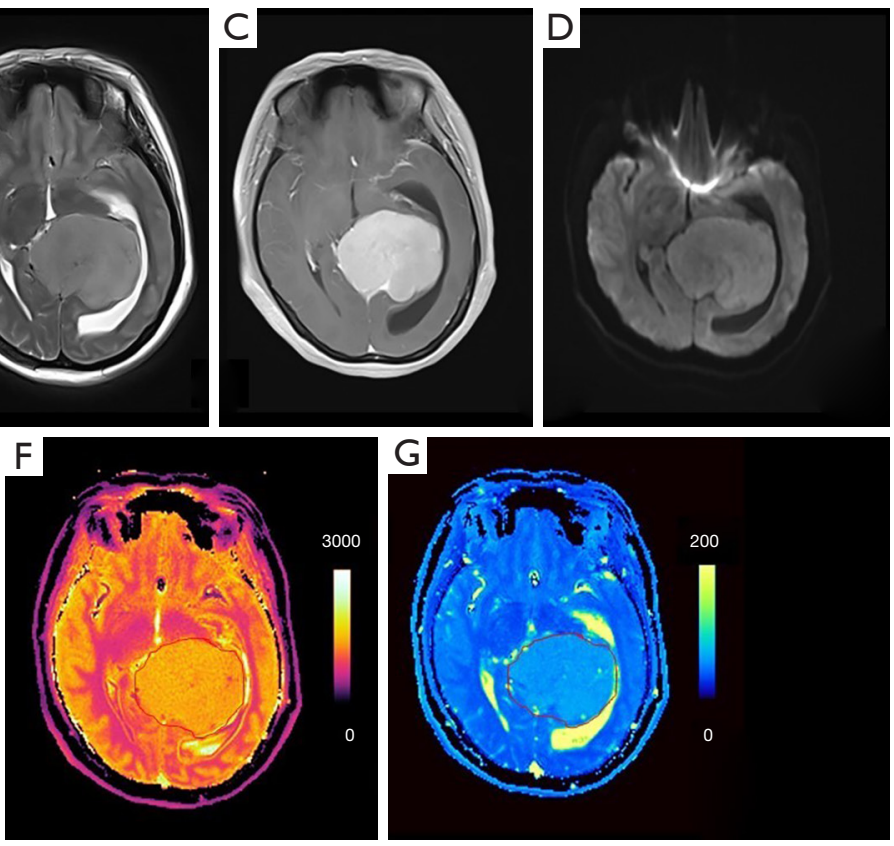

Figure 4 Data from a representative 44-year-old female patient with fibrous meningioma. (A) $\mathrm{T}_{1}$-weighted imaging (T1WI) MRI; (B) $\mathrm{T}_{2}{ }^{-}$ weighted imaging (T2WI) MRI; (C) contrast-enhanced T1WI; (D) diffusion-weighted imaging (DWI); (E) apparent diffusion coefficient map; (F) T1 value derived from magnetic resonance fingerprinting (MRF); (G) T2 value derived from MRF.
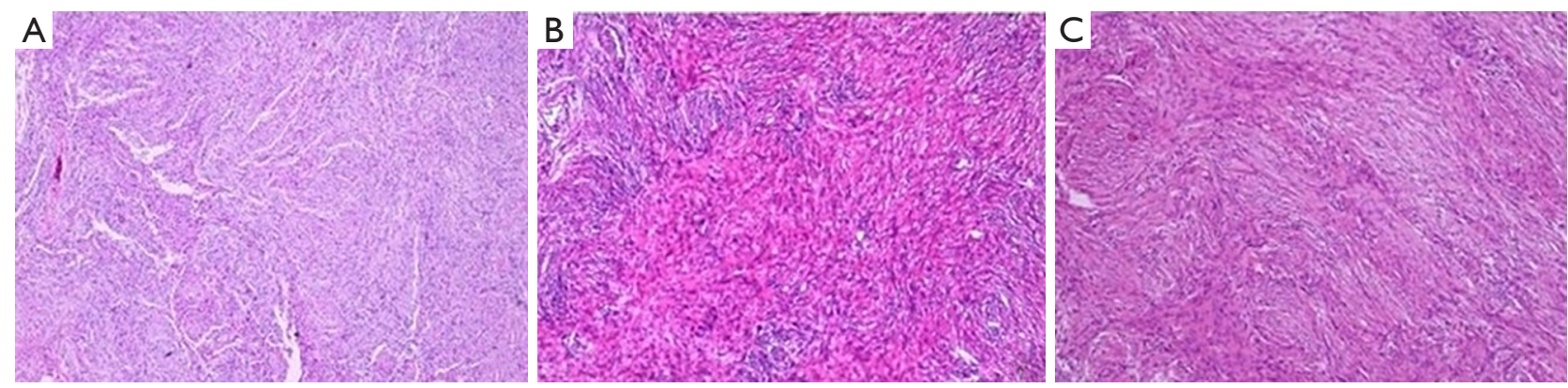

Figure 5 Pathological results of a representative patient with meningothelial meningioma (A), a representative patient with transitional meningioma (B), and a representative patient with fibrous meningioma (C). [A,C: hematoxylin-eosin (HE) stain, 4×10; B: HE stain, 10×10].

The AUCs for differentiating between the meningothelial and transitional groups for the T1, T2, and combined $\mathrm{T} 1$ and $\mathrm{T} 2$ values were $0.819,0.822$, and 0.826 , respectively. The $\mathrm{T} 1$ and $\mathrm{T} 2$ values both had a sensitivity of $80 \%$ and a specificity of $83.33 \%$, with an optimal threshold of 1564 and 72, respectively. The combination of the T1 and $\mathrm{T} 2$ values also had a sensitivity of $80 \%$ and a specificity of $83.33 \%$. For the meningothelial and fibrous groups, the AUCs for the T1, T2, and combined T1 and T2 values were $0.841,0.874$, and 0.903 , respectively. The optimal threshold for the $\mathrm{T} 1$ value was 1,563 , with a sensitivity of $80 \%$ and a specificity of $84.62 \%$, whereas for the T2 value, the optimal threshold was 71 and the sensitivity and specificity were $86.67 \%$ and $84.62 \%$, respectively. The combination of the $\mathrm{T} 1$ and $\mathrm{T} 2$ values had a sensitivity of $80 \%$ and a specificity of $92.31 \%$.

\section{Discussion}

Our results indicated transitional and fibrous meningiomas 

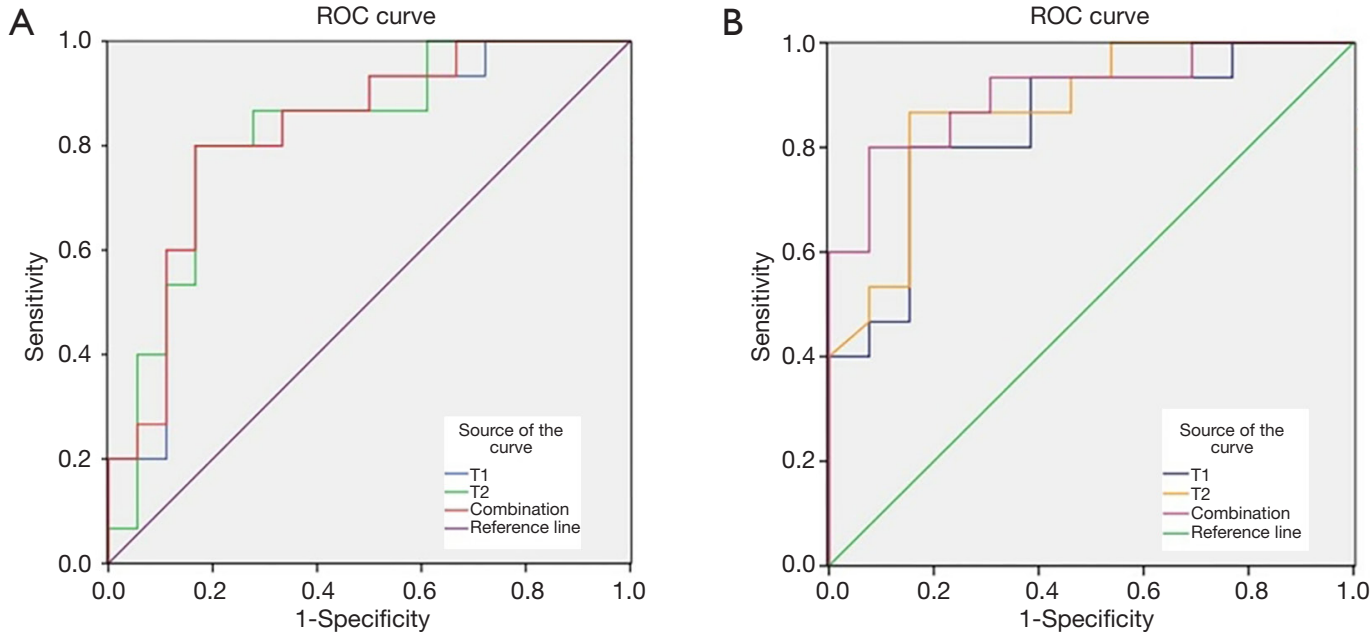

Figure 6 (A) Receiver-operating characteristic (ROC) curve of T1 and T2 values and the combination of the T1 and T2 values (combined variable) for the differential diagnosis of (A) meningothelial and transitional meningiomas, (B) meningothelial and fibrous meningiomas.

to have significantly lower T1 and T2 values than meningothelial meningiomas. However, conventional MRI, including T1WI, T2WI, contrast-enhanced T1WI, and ADC values, indicated no significant differences between transitional/fibrous meningiomas and meningothelial meningiomas. The ROC analyses showed the T1 and T2 mapping generated by MRF may have potential for differentiating transitional and fibrous meningiomas from meningothelial meningiomas.

In the present study, we compared and analyzed the value of conventional MRI and DWI techniques in the classification of meningiomas. For the analysis using conventional T1WI, T2WI, and contrast-enhanced T1WI, neither radiologist was able to differentiate transitional and fibrous meningiomas from meningothelial meningiomas. The visual observation of conventional MRI had larger overlaps between the transitional and fibrous meningiomas and the meningothelial meningiomas. Further, the ADC values did not differ statistically between the three meningioma subtypes, a finding which was consistent with the results of a previous study in another lab (20). High cellularity in meningothelial meningiomas leads to a decrease in ADC values, and the rich fibrous nature of transitional and fibrous meningiomas has similar effects, which may account for the lack of statistically significant difference in the ADC values of these meningioma subtypes in this study.

Current research of the liver (21), prostate (22), musculoskeletal system, cardiovascular system (23), and other organ systems shows that MRF has the advantage of yielding rapid multi-parameter results compared with traditional MRI methods, and it is able to quantitatively and reproducibly measure tissue characteristics. In view of these advantages, this technology has the potential to become a useful tool for MRI. Furthermore, a previous study by $\mathrm{Li}$ et al. provided evidence that MRF has the potential to accurately distinguish brain tissue components and detect local micro-regional lesions (24). Our results support these findings, and also indicate that the quantitative advantages of MRF allow signal digitization and utilization of values to directly and objectively reflect the difference between different meningioma subtypes.

The T1 and T2 values yielded from MRF were able to differentiate WHO grade I transitional and fibrous meningiomas from meningothelial meningiomas, which may be related to the histology of brain tumors. The $\mathrm{T} 1$ and $\mathrm{T} 2$ values in tissue depend on many factors, such as structure, cellular density, water content, and collagen fibers. In meningothelial meningiomas, tumor cells are similar to normal arachnoid cap cells, which are surrounded by a thin collagen membrane to form leaflets. The sparse fibrous stroma is mainly limited to the trabecula that can divide the tumor into lobules with high water content, which increases the $\mathrm{T} 1$ and $\mathrm{T} 2$ values. In contrast to meningothelial meningiomas, the tumor cells in transitional and fibrous meningiomas are denser and are arranged into a compact structure. The tumor cells are also separated by a large number of collagen fibers. 
The dense cellularity and abundant collagen fibers of transitional and fibrous meningiomas may decrease the $\mathrm{T} 1$ and $\mathrm{T} 2$ values. Therefore, these differences in the histological characteristics between transitional and fibrous meningiomas and meningothelial meningiomas may contribute to differences in $\mathrm{T} 1$ and $\mathrm{T} 2$ values. $\mathrm{T} 1$ and $\mathrm{T} 2$ values did not vary significantly between transitional and fibrous meningiomas in this study, which could be due the similar pathological characteristics of these meningiomas.

Even with similar treatments, different WHO grade I meningioma subtypes may have different outcomes. Hemorrhage during meningioma surgery often results in a poor prognosis. Meningothelial meningiomas are rarely associated with intraoperative hemorrhage in surgery, whereas transitional and fibrous meningiomas have a higher tendency to hemorrhage. Moreover, the $22 \mathrm{q}$ chromosomal abnormality is related to tumor aggressiveness. This chromosomal abnormality is more commonly seen in both the transitional and fibrous subtypes than in meningothelial meningiomas (25). Transitional and fibrous meningiomas are highly aggressive, which may be the reason why intraoperative hemorrhage is more common than in meningothelial meningiomas. Therefore, the differentiation of transitional and fibrous meningiomas from meningothelial meningiomas using a non-invasive technique, such as MRF, before surgery could contribute to preoperative planning and a more accurate prognosis.

The present study has some limitations, such as a small sample size, the exclusion of other meningioma subtypes, and a lack of grading studies. Due to the small sample size, even a small number of outliers may have significantly affected the results of the study. Therefore, in-depth research that appropriately expands the study sample inclusion criteria and enrolls patients with other meningioma subtypes will ensure greater accuracy, comprehensiveness, and generalizability of the outcomes. Second, additional correlation analyses between pathological tumor markers and T1 and T2 values should be conducted in the future to support the findings of the present study.

\section{Conclusions}

The T1 and T2 values yielded from MRF can differentiate transitional and fibrous meningiomas from meningothelial meningiomas. The combination of $\mathrm{T} 1$ and $\mathrm{T} 2$ values achieved the best diagnostic performance for differentiating transitional from meningothelial meningiomas (AUC
$=0.826$, sensitivity $=80 \%$, and specificity $=83.33 \%)$ and differentiating fibrous from meningothelial meningiomas (AUC $=0.903$, sensitivity $=80 \%$, and specificity $=92.31 \%$ ). These findings could benefit preoperative treatment options for meningiomas and allow for more accurate prognoses.

\section{Acknowledgments}

Funding: This work was supported by the National Key R\&D Program of China (No. 2017YFE0103600), National Natural Science Foundation of China (No. 81720108021), Zhongyuan Thousand Talents Plan Project-Basic Research Leader Talent (No. ZYQR201810117), Scientific and Technological Research Project of Henan Province (No. 182102310496), and Medical Science and Technology Research Project of Henan Province (No. 2018020403).

\section{Footnote}

Conflicts of Interest: All authors have completed the ICMJE uniform disclosure form (available at http:// dx.doi.org/10.21037/qims-20-732). XZ, MN, and GK are employees of Siemens Healthcare. The other authors have no conflicts of interest to declare.

Ethical Statement: The present prospective study was approved by our hospital's institutional ethics committee. All enrolled patients provided signed informed consent.

Open Access Statement: This is an Open Access article distributed in accordance with the Creative Commons Attribution-NonCommercial-NoDerivs 4.0 International License (CC BY-NC-ND 4.0), which permits the noncommercial replication and distribution of the article with the strict proviso that no changes or edits are made and the original work is properly cited (including links to both the formal publication through the relevant DOI and the license). See: https://creativecommons.org/licenses/by-nc-nd/4.0/.

\section{References}

1. O'Leary S, Adams WM, Parrish RW, Mukonoweshuro W. Atypical imaging appearances of intracranial meningiomas. Clin Radiol 2007;62:10-7.

2. Villa C, Miquel C, Mosses D, Bernier M, Stefano AL. The 2016 World Health Organization classification of tumours of the central nervous system. Presse Med 2018;47:e187-200. 
3. Kunimatsu A, Kunimatsu N, Kamiya K, Katsura M, Mori H, Ohtomo K. Variants of meningiomas: a review of imaging findings and clinical features. Jpn J Radiol 2016;34:459-69.

4. Gajbhiye S, Gosal JS, Pandey S, Das KK. Apoplexy Inside a Giant Medial Sphenoid Wing Meningothelial (Grade I) Meningioma: An Extremely Rare but a Potentially Dangerous Complication. Asian J Neurosurg 2019;14:961-3.

5. Pavlisa G, Rados M, Pazanin L, Padovan RS, Ozretic D, Pavlisa G. Characteristics of typical and atypical meningiomas on ADC maps with respect to schwannomas. Clin Imaging 2008;32:22-7.

6. Watanabe Y, Yamasaki F, Kajiwara Y, Takayasu T, Nosaka R, Akiyama Y, Sugiyama K, Kurisu K. Preoperative histological grading of meningiomas using apparent diffusion coefficient at 3T MRI. Eur J Radiol 2013;82:658-63.

7. Giraudo C, Cavaliere A, Lupi A, Guglielmi G, Quaia E. Established paths and new avenues: a review of the main radiological techniques for investigating sarcopenia. Quant Imaging Med Surg 2020;10:1602-13.

8. Shankar JJS, Hodgson L, Sinha N. Diffusion weighted imaging may help differentiate intracranial hemangiopericytoma from meningioma. J Neuroradiol 2019;46:263-7.

9. She D, Liu J, Zeng Z, Xing Z, Cao D. Diagnostic accuracy of diffusion weighted imaging for differentiation of supratentorial pilocytic astrocytoma and pleomorphic xanthoastrocytoma. Neuroradiology 2018;60:725-33.

10. Yiping L, Kawai S, Jianbo W, Li L, Daoying G, Bo Y. Evaluation parameters between intra-voxel incoherent motion and diffusion-weighted imaging in grading and differentiating histological subtypes of meningioma: A prospective pilot study. J Neurol Sci 2017;372:60-9.

11. Ma D, Gulani V, Seiberlich N, Liu K, Sunshine JL, Duerk JL, Griswold MA. Magnetic resonance fingerprinting. Nature 2013;495:187-92.

12. Körzdörfer G, Kirsch R, Liu K, Pfeuffer J, Hensel B, Jiang Y, Ma D, Gratz M, Bär P, Bogner W, Springer E, Cardoso PL, Umutlu L, Trattnig S, Griswold M, Gulani V, Nittka M. Reproducibility and Repeatability of MR Fingerprinting Relaxometry in the Human Brain. Radiology 2019;292:429-37.

13. Pan S, Wang XQ, Guo QY. Quantitative assessment of hepatic fibrosis in chronic hepatitis B and C: T1 mapping on Gd-EOB-DTPA-enhanced liver magnetic resonance imaging. World J Gastroenterol 2018;24:2024-35.

14. Hamilton-Craig CR, Strudwick MW, Galloway GJ. T1 Mapping for Myocardial Fibrosis by Cardiac Magnetic Resonance Relaxometry-A Comprehensive Technical Review. Front Cardiovasc Med 2017;3:49.

15. Panda A, Mehta BB, Coppo S, Jiang Y, Ma D, Seiberlich N, Griswold MA, Gulani, V. Magnetic Resonance Fingerprinting-An Overview. Curr Opin Biomed Eng 2017;3:56-66.

16. Xie J, Lyu M, Zhang K, Hui ES, Wu EX, Wang Z. Fast dictionary generation and searching for magnetic resonance fingerprinting. Annu Int Conf IEEE Eng Med Biol Soc 2017;2017:3256-9.

17. Badve C, Yu A, Dastmalchian S, Rogers M, Ma D, Jiang Y, Margevicius S, Pahwa S, Lu Z, Schluchter M, Sunshine J, Griswold M, Sloan A, Gulani V. MR Fingerprinting of Adult Brain Tumors: Initial Experience. AJNR Am J Neuroradiol 2017;38:492-9.

18. MacKay AL, Vavasour IM, Rauscher A, Kolind SH, Mädler B, Moore GR, Traboulsee AL, Li DK, Laule C. $\mathrm{MR}$ relaxation in multiple sclerosis. Neuroimaging Clin $\mathrm{N}$ Am 2009;19:1-26.

19. Prayer D. MR Fingerprinting: An Advance for Patients with Temporal Lobe Epilepsy. Radiology 2018;288:813-4.

20. Sanverdi SE, Ozgen B, Oguz KK, Mut M, Dolgun A, Soylemezoglu F, Cila A. Is diffusion-weighted imaging useful in grading and differentiating histopathological subtypes of meningiomas?. Eur J Radiol 2012;81:2389-95.

21. Kim KA, Park MS, Kim IS, Kiefer B, Chung W, Kim M, Kim KW. Quantitative evaluation of liver cirrhosis using $\mathrm{T} 1$ relaxation time with 3 tesla MRI before and after oxygen inhalation. J Magn Reson Imaging 2012;36:405-10.

22. Yu AC, Badve C, Ponsky LE, Pahwa S, Dastmalchian S, Rogers M, Jiang Y, Margevicius S, Schluchter M, Tabayoyong W, Abouassaly R, McGivney D, Griswold MA, Gulani V. Development of a Combined MR Fingerprinting and Diffusion Examination for Prostate Cancer. Radiology 2017;283:729-38.

23. Hamilton JI, Jiang Y, Chen Y, Ma D, Lo WC, Griswold MA, Seiberlich N. MR fingerprinting for rapid quantification of myocardial T1,T2, and proton spin density. Magn Reson Med 2017;77:1446-58.

24. Li M, Dong ZC, Zhang XW, Zhang XM. The technique of magnetic resonance fingerprinting and its latest development (in Chinese). Sci Sin Vitae 
2019;49:28-40.

25. Zikou A, Alexiou GA, Goussia A, Kosta P, Xydis VG, Voulgaris S, Kyritsis AP, Argyropoulou MI. The role

Cite this article as: Zhang R, Shen Y, Bai Y, Zhang X, Wei W, Lin R, Feng Q, Wang M, Zhang M, Nittka M, Koerzdoerfer G, Wang M. Application of magnetic resonance fingerprinting to differentiate grade I transitional and fibrous meningiomas from meningothelial meningiomas. Quant Imaging Med Surg 2021;11(4):1447-1457. doi: 10.21037/qims-20-732 of diffusion tensor imaging and dynamic susceptibility perfusion MRI in the evaluation of meningioma grade and subtype. Clin Neurol Neurosurg 2016;146:109-15. 